\title{
Intermédialités
}

Histoire et théorie des arts, des lettres et des techniques

Intermediality

History and Theory of the Arts, Literature and Technologies

\section{Des problématiques de présentation de l'art vidéo à l'agentivité créatrice : sous-titrage et articulation des temps chez Anri Sala et Vera Frenkel}

\section{Maude Lefebvre}

Numéro 27, printemps 2016

traduire

translating

URI : https://id.erudit.org/iderudit/1039813ar

DOI : https://doi.org/10.7202/1039813ar

Aller au sommaire du numéro

Éditeur(s)

Revue intermédialités (Presses de l’Université de Montréal)

ISSN

1920-3136 (numérique)

Découvrir la revue

Citer cet article

Lefebvre, M. (2016). Des problématiques de présentation de l'art vidéo à

l'agentivité créatrice : sous-titrage et articulation des temps chez Anri Sala et

Vera Frenkel. Intermédialités / Intermediality, (27).

https://doi.org/10.7202/1039813ar
Résumé de l'article

L'exposition de l'art vidéo en contexte muséal donne souvent lieu à des problématiques liées entre autres à la question du sous-titrage dans un but de diffusion multilingue. L'utilisation d'un tel moyen technique occasionne dans bien des cas des pertes de sens importantes, voire l'effacement total de la dimension sonore, mais peut également produire un décloisonnement des médiums et une simultanéité des expériences. Certains artistes utilisent quant à eux le sous-titrage comme matériau artistique à part entière, comme la Canadienne d'origine slovaque Vera Frenkel et l'Albanais Anri Sala. 


\title{
Des problématiques de présentation de l'art vidéo à l'agentivité créatrice : sous-titrage et articulation des temps chez Anri Sala et Vera Frenkel
}

\author{
MAUde LeFEBVRE
}

e sous-titrage comme mode de réception de l'art vidéo est source de nombreux défis chez les artistes et les commissaires. Bien souvent, des œuvres vidéographiques sont présentées dans une langue que le public du lieu d'exposition ne connaît pas, et le contact avec l'œuvre passe alors obligatoirement par le sous-titrage qui devient le seul moyen de réception du contenu oral. Il arrive aussi que des œuvres vidéo, accolées les unes aux autres, soient exposées sans leur bande sonore pour éviter la contamination sonore : le sous-titrage permet alors de remédier à la suppression du son. Ainsi, au printemps 20I4, au Museum of Modern Art de New York se tenait une rétrospective des travaux de l'artiste brésilienne Lygia Clark ${ }^{1}$. Dans la première partie de l'exposition, les commissaires avaient regroupé plusieurs œuvres performatives ou relationnelles de Clark sous la forme de documents vidéo projetés au mur, entrecoupés dans l'espace physique par des objets utilisés lors des performances originales. Fait étonnant, toutefois : plusieurs des vidéos étaient présentées sans leur piste audio, alors entièrement substituée par le sous-titrage des propos tenus par les intervenants. Ici, les contraintes muséographiques ont conduit à un effacement total de la dimension sonore, et l'expérience de l'œuvre vidéo sous-titrée s'est trouvée modifiée par sa « traduction » du son en texte. ““”

Inversement, dans le cadre de la biennale 2010 du Whitney Museum of American Art de New York, plusieurs vidéos avaient également été sous-titrées et mises en sourdine, mais on les avait munies d'écouteurs à l'attention du spectateur soucieux d'une expérience plus complète. L'intégration de l'œuvre au sous-texte de l'exposition était d'autant plus facilitée que le spectateur, à même de circuler librement dans l'espace

I On trouve le dossier de l'exposition sur le site du MoMA: «Lygia Clark: The Abandonment of Art, 1948-1988 », The Museum of Modern Art, du Io mai au 24 août 2014, https://www.moma.org/calendar/exhibitions/I422?locale=en (consultation le I9 février 2017). 
du musée et de s'attarder devant l'œuvre vidéo de la même façon que devant un tableau, n'était pas contraint de se couper momentanément du fil narratif par son entrée dans une salle de type «black box ». Il apparaît donc que de nouvelles possibilités discursives émergent de cette façon d'exposer l'art vidéo sans sa dimension sonore, puisque le sous-titrage permet un décloisonnement des médiums² de même qu'une simultanéité des expériences. Les autres types d'art peuvent ainsi profiter de cette relation de proximité avec la vidéo et entrer en dialogue avec elle sans craindre d'être contaminés, voire étouffés par le son.

Des problématiques liées à la traduction émergent aussi fréquemment de la pratique du sous-titrage des œuvres vidéo. En effet, les occurrences de propos lost in translation $^{3}$ ne sont pas rares. Le spectateur, qui n'a alors accès qu'à une partie de la signification, se retrouve privé des subtilités de plus ou moins grande importance désirées par le créateur. Toutefois, certains vidéastes ont recours au sous-titrage non pas à des fins strictement utilitaires et dans un but de diffusion multilingue, mais plutôt comme choix artistique à part entière. C'est notamment le cas de la Canadienne d'origine slovaque Vera Frenkel et de l'Albanais Anri Sala, qui ont tous deux fait du sous-titrage un outil artistique de première importance dans ... du transitbar (1992) et Intervista (Finding the Words)(1999). Par cette transformation de la fonction utilitaire du sous-titrage en un vecteur d'opérativité créatrice, ces deux propositions permettent une articulation des temps complexe entre passé, présent et futur, et mettent de l'avant des questions liées à la mémoire, à la transmission et à la réécriture d'une histoire personnelle ou collective.

« Puisque la traduction existe, il faut bien qu'elle soit possible ${ }^{4}$ », écrivait Paul Ricour, qui écartait ainsi involontairement de sa sphère de considération bon nombre d'œuvres jouant sur l'impossibilité de la traduction ou sur des décalages qui affectent la compréhension. Chez Frenkel et Sala, la traduction est en effet possible, mais elle induit inévitablement une perte de sens et une confrontation au passé. Ce qui, au départ, apparaît comme simple moyen technique, à savoir le sous-titrage de l'art vidéo, révèle des problématiques plus spécifiquement liées aux études exiliques et portant sur la

${ }^{2}$ Du fait que l'œuvre vidéo est ainsi présentée sans risque de contamination sonore de ses voisines, on peut envisager que dans un même espace cohabitent œuvres bidimensionnelles - peinture, photographie, dessin, art imprimé, entre autres —, œuvres tridimensionnelles, installations, créations sonores avec ou sans écouteurs, performance, danse, musique devant public et bien d'autres types d'art.

${ }^{3}$ Eva Hoffman, Lost in Translation: A Life in a New Language [1989], Londres, Penguin Books, 1990 .

4 Paul Ricour, Sur la traduction, Paris, Bayard, 2003, p. 29. 
mémoire. Alors que l'amorce du présent texte a été résolument muséologique, notre analyse nous conduira à transcender les questions techniques relatives à l'exposition de l'art en général.

\section{PRÉSENT TRANSITOIRE ET PROCESSUS MÉMORIEL CHEZ VERA FRENKEL}

L'installation vidéo ... du transitbar (1992) de Vera Frenkel a été présentée pour la première fois à la documenta IX de Cassel. Investissant tout l'espace de la pièce lui étant réservée dans le cadre de cet évènement international, Frenkel met en place un véritable espace de détente reprenant tous les codes du piano-bar : elle y installe des tables; un piano dont jouera un pianiste invité ou qui sera activé mécaniquement; un bar où l'on peut commander des boissons alcoolisées à un barman, parfois même à l'artiste elle-mêmes; des éditions d'un journal créé spécifiquement pour l'occasion; quelques plantes et autres accessoires de décoration. Dans cet espace convivial, toutefois, Frenkel conçoit une installation disque laser à six canaux, se présentant sous la forme de six petits téléviseurs disséminés dans l'espace du piano-bar, encastrés dans les murs ou déposés sur le mobilier. Par l'entremise de ces téléviseurs, l'artiste présente les témoignages de quatorze exilés ou déportés — des amis de Frenkel — provenant de différents contextes et de différentes régions du monde, et éprouvant une difficulté d'appartenance à une communauté ou une difficulté à trouver leur identité entre plusieurs cultures. Le spectateur est donc invité à se détendre au bar, à siroter un verre de whisky ou de vodka, et à s'imprégner de ces histoires de déracinement racontées de façon très personnelle, intime, dans un plan de caméra rapproché où le regard de l'intervenant est constamment fixé sur le sien.

La transmission de l'histoire de ces personnes se complique cependant parce que leur témoignage a été doublé dans une langue que Vera Frenkel qualifie de marginalisée ou dominée - soit le yiddish ou le polonais - et sous-titrée dans une

s L'artiste servit notamment des boissons aux visiteurs chaque jour de cette première présentation à la documenta IX de Cassel, soit du 13 juin au 20 septembre 1992. Vera Frenkel, « from the Transit Bar », Public, vol. 9, 1994, p. 80-8I. Jean Gagnon, Lydia Haustein, Jeanne Randolph et Irit Rogoff, Vera Frenkel: ... from the Transit Bar/... du transitbar, Toronto, Power Plant Contemporary Art Gallery et National Gallery of Canada, 1994, p. 139. Elle le fit également le dernier soir de la présentation à la National Gallery of Canada, le I7 août 2014. Natasha Chaykowski, « Transient Spaces: Empathy in Vera Frenkel's Transit Bar », Canadian Art, 22 août 20I4, http://canadianart.ca/reviews/vera-frenkel-from-the-transit-bar (consultation le I2 janvier 20I7). 
langue impérialiste ou dominante - soit le français (voir la figure I), l'anglais (voir la figure 2) ou l'allemand (voir la figure 3), en alternance.
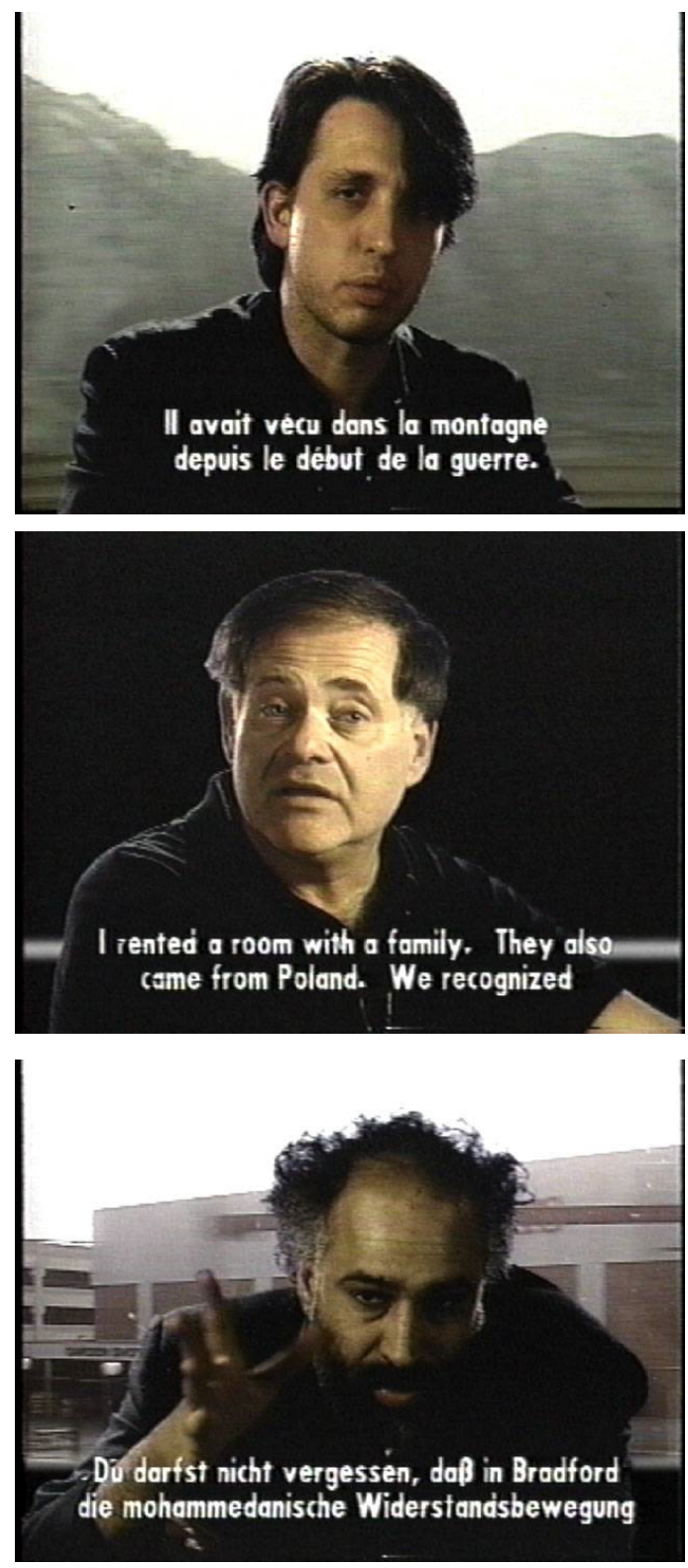

Fig. I-3. Vera Frenkel, ... du transitbar (témoignage de Basil, Bernie et Julian), installation disque laser à six canaux, 1992.

(c) Vera Frenkel 
En effet, au cours d'un seul et même témoignage, une « cellule ${ }^{6} »$ de sous-titrage - une phrase ou une partie de phrase, selon le cas - a été traduite en français, la suivante en anglais, l'autre en allemand, et ainsi de suite. Si bien que le sous-titrage ne permet qu'une compréhension partielle du récit d'un intervenant : pour en suivre le fil conducteur, il faut impérativement savoir lire les trois langues. Par ce procédé d'alternance des langues dans la traduction, sous-titrée, des récits oraux, Vera Frenkel a voulu créer une perte de sens et mettre le spectateur dans la position de l'étranger, de la personne marginalisée qui se retrouve forcée d'habiter un pays où sa langue maternelle n'est pas dominante, voire pas du tout parlée. Faire l'expérience de l'œuvre de Frenkel, c'est également faire l'expérience d'une perte de sens et d'une défaillance linguistique.

Il faut également savoir que ces témoignages sont performés plusieurs années après les faits relatés, les intervenants faisant appel à leur mémoire afin de les raconter le plus fidèlement possible. Cet intervalle entre le temps de l'évènement et le temps de son évocation, qu'il ait été ou non parsemé d'autres épisodes de témoignage, est d'ailleurs palpable chez les personnes filmées par Frenkel. La parole est parsemée d'hésitations et d'oublis, effets inhérents au travail de mémoire, mais plus encore à ce que Ricœur appelle la «mémoire empêchée7 », qu'il définit comme la difficulté à se souvenir d'un épisode traumatique. La reconstitution mentale de l'évènement peut ainsi s'avérer ardue pour le visiteur de ... du transitbar, qui ne saisit que des bribes de l'histoire, ce qui le place en situation d'incompréhension. De plus, comme l'explique Jean Gagnon dans le catalogue des versions torontoise et ottavienne de l'œuvre présentées en 1995 , « [u] ne ambiguïté persiste quant à la véracité des choses dites et à la sincérité des visages qui nous parlent, tout comme un jeu persiste dans l'endossement de signes identifiables à des rôles et à des voix empruntées ${ }^{8} \gg$. En effet, pour ajouter à cette difficulté de saisir la totalité de l'histoire, un important décalage est occasionné par le doublage en yiddish ou en polonais des quatorze récits, comme si la personne à l'écran du téléviseur était désincarnée ou jouait un rôle, comme si elle n'était pas le véritable témoin de sa propre histoire. Par exemple, l'histoire racontée par Julian, un Afro-Américain (voir la figure 3), nous parvient par l'intermédiaire d'une voix

${ }^{6} \mathrm{La}$ « cellule » en sous-titrage correspond généralement à un temps d'exposition de deux secondes environ et peut parfois contenir une phrase complète. Toutefois, dans le cas d'une phrase plutôt longue, celle-ci doit être découpée en plusieurs cellules successives de deux ou trois lignes chacune.

7 Paul Ricour, La mémoire, l'bistoire, l'oubli, Paris, Seuil, 200o, p. 83.

${ }^{8}$ Gagnon et al., I994, p. 2 I. 
s'exprimant en polonais, alors que les sous-titres sont en français. Le témoignage en apparence direct, intime, devient alors plus distant. L'œuvre ... du transitbar de Vera Frenkel fait donc en sorte d'installer une étrangeté dans le témoignage, mais également, comme nous le verrons dans les prochaines pages, de brouiller la limite entre présent, passé et futur par ce jeu complexe relatif au sous-titrage.

Chez Vera Frenkel, le présent est un présent transitoire. En effet, le piano-bar construit in situ par l'artiste peut s'aborder comme un espace en mouvement, qui aurait quitté son lieu d'origine sans être encore parvenu à destination. Pour Frenkel, l'espace physique qu'elle bâtit de toutes pièces dans ce lieu d'exposition de Cassel est le symbole d'un transit entre le passé, ou le lieu d'origine des intervenants, et leur futur dans un pays étranger, choisi ou imposé.

Mais le présent est aussi une question d'oralité chez Frenkel. Dans ce piano-bar, des situations de rencontre réelles se créent entre les spectateurs, devenus clients de l'établissement temporaire9. Épuisés ou excités par une journée passée à explorer les propositions artistiques de la documenta IX, ces spectateurs arrêtent au transitbar de Frenkel pour se poser un moment, pour relaxer entre amis autour d'un verre. Les discussions sont variées, sérieuses ou légères, enflammées ou contenues. En ce sens, le transitbar diffère peu des bars que l'on retrouve partout à travers le monde. Là s'échange une parole directe, instantanée, résolument ancrée dans le présent. Mais l'oralité ne se manifeste pas que dans ce déliage des langues que permet l'espace du piano-bar. Les vidéos installées aux quatre coins de la pièce sont également conçues de façon à mettre de l'avant une oralité médiatique de témoignage. Les histoires sont toutes racontées en misant sur le rapport à l'autre, à la personne qui dans le bar reçoit le témoignage, presque comme une confession. Les intervenants sont filmés en plan rapproché, leur visage tourné vers la caméra, le regard plongé dans celui, hypothétique, du spectateur (voir la figure 4). Frenkel crée une intimité médiatisée, par laquelle devrait s'opérer une transmission de la mémoire personnelle. Mais même si le rapport entre l'intervenant et le spectateur est assez direct, un décalage est créé par l'utilisation du sous-titrage et du doublage. En ne comprenant pas tout ce qui est raconté, le spectateur reçoit un récit incomplet, fragmenté. Ce dernier est également dans l'impossibilité de lui raconter sa propre expérience en retour, la seule option pour ouvrir le dialogue étant de la partager avec les autres clients du bar.

\footnotetext{
9 Ibid., p. 2O-2I.
} 


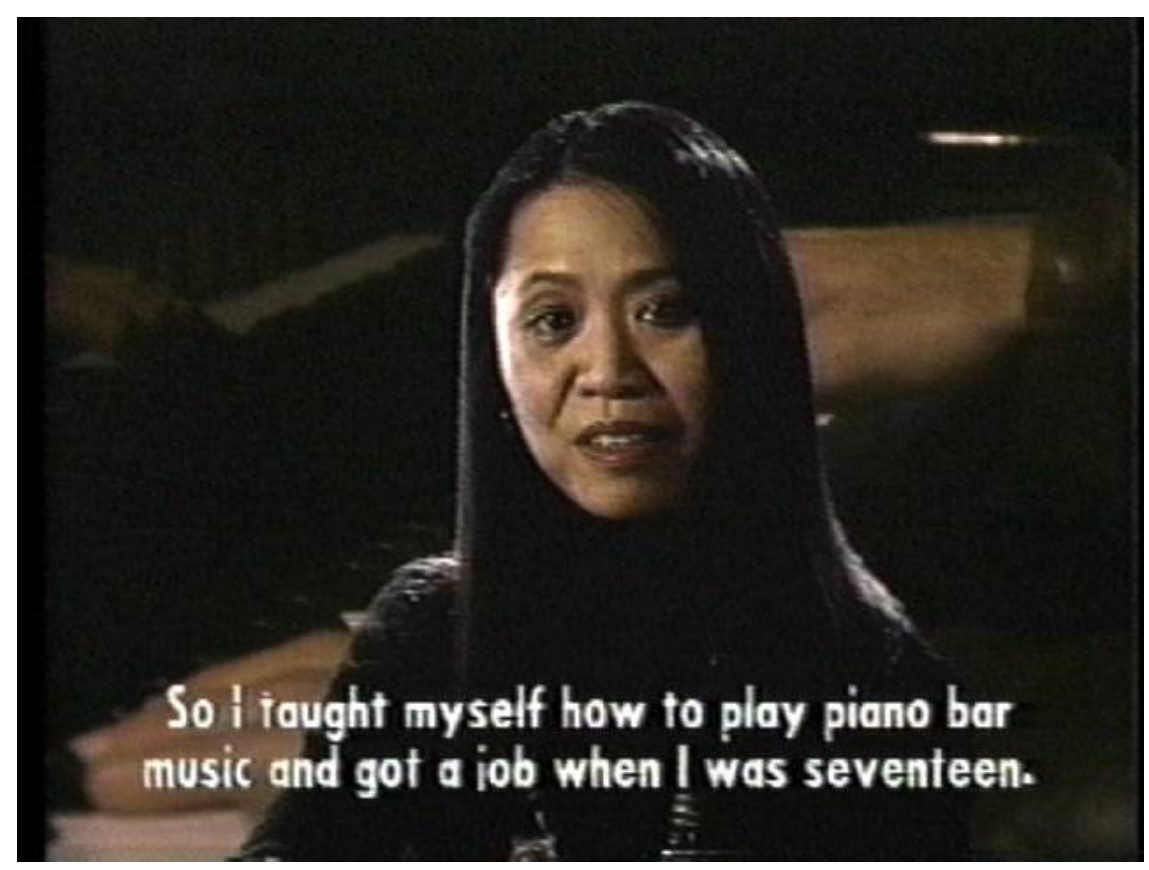

Fig. 4. Vera Frenkel, ... du transitbar (témoignage d'Alexina), installation disque laser à six canaux, 1992. (C) Vera Frenkel

De ce fait, il se crée dans l'espace du piano-bar de Frenkel une sorte d'enchevêtrement narratif ${ }^{\circ}$, pour reprendre le terme employé par Paul Ricœur, qui provoque inévitablement une contamination entre les histoires racontées par les personnes médiatisées et celles racontées par les visiteurs. Il y a alors un échange de récits et de conversations qui «structurent et occupent le présent ${ }^{\mathrm{II}} \gg$. Dans ce même lieu se déploie un présent de la mémoire personnelle qui, par cet enchevêtrement narratif, devient un présent historique commun. Tous ces récits s'articulent dans un même présent et forment une sorte de narration collective, en rupture avec la «narration nationale » ou «l'histoire officielle ». À ce propos, l'auteure Adair Rounthwaite explique en quoi l'œuvre de Frenkel joue à la fois sur le terrain de l'histoire individuelle et de l'histoire collective :

Bien qu'un des narrateurs raconte une histoire directement liée à l'Holocauste, la majorité relatent des histoires d'immigration sans lien géographique ou politique direct

\footnotetext{
Io Paul Ricœur, Temps et récit [1983], t. I, Paris, Seuil, I99I.

II Gagnon (dir.), 1994, p. 22.
} 
avec cet évènement. En présentant ces histoires à l'aide d'indices visuels et auditifs liés à l'Holocauste [le choix des langues, notamment], les vidéos réactivent la tendance occidentale à l'envisager comme le génocide des génocides. [...] Cela peut mettre en lumière la gravité d'autres conflits mondiaux fréquemment ignorés de la population et des gouvernements occidentaux, mais peut aussi renforcer un point de vue eurocentrique du génocide qui tend à rendre ces conflits invisibles ${ }^{12}$.

Par ce rappel constant à l'Holocauste, notamment par le choix des langues comme le yiddish ou le polonais, Frenkel fait effectivement en sorte d'inscrire la petite histoire de chacun dans la «grande histoire » et dénonce cette tendance à universaliser le témoignage en le privant de ses référents réels, opération pouvant vider le discours de sa signification. Pour le visiteur, cela ajoute au sentiment d'étrangeté, voire d'incompatibilité, puisque ce qu'il pense comprendre et, dans une certaine mesure, partager avec la personne médiatisée, selon ses connaissances de l'évènement relaté, se retrouve dilué dans un discours universalisant désengageant. Frenkel réussit donc à accentuer le décalage déjà ressenti par l'inadéquation des sous-titres à la parole des intervenants.

Nous comprenons dès lors que le piano-bar de Vera Frenkel est un espace ancré dans le présent, un présent transitoire, entre deux lieux et entre deux temporalités. Nous comprenons également que ce présent est un présent de l'oralité, du récit direct, et la source d'un « enchevêtrement narratif » par la contamination des histoires. Mais l'œuvre de Vera Frenkel implique aussi une forte dimension mémorielle, un rapport au passé s'effectuant par la mémoire. En effet, cette oralité du témoignage que nous avons abordée précédemment, bien ancrée dans le présent, permet aussi de faire intervenir la mémoire et l'histoire. En faisant l'expérience de l'œuvre de Frenkel et en s'imprégnant des quatorze témoignages, le spectateur accède aux souvenirs déformés par le temps des intervenants. Dans ce cas, le but n'est pas de retrouver ce qui est perdu et de « compléter les trous de l'histoire », mais plutôt d'accoler tous les récits par un processus d'enchevêtrement et d'accéder à « la réalité par recouvrement des histoires relatées de chacun ${ }^{13} \gg$. On réactualise donc le passé, mais toujours en insistant sur la perte qui est inévitable dans un processus mémoriel. Cette insistance sur celle-ci est d'ailleurs renforcée par l'idée que le passé est contenu et compressé par le sous-titrage des langues marginalisées en langues dominantes. Il y a là une perte de sens occasionnée

\footnotetext{
${ }^{12}$ Adair Rounthwaite, « Community and alienation in Vera Frenkel's ... from the Transit Bar », Beyond the Object (conférence), Toronto, Université York, 7 avril 2005, notre traduction.

I3 Gagnon et al., 1994, p. 2I.
} 
par la traduction, ce qui, pour Paul Ricœur, fait partie de la tâche du traducteur, où il est « procédé à un certain sauvetage et à un certain consentement à la perte ${ }^{\mathrm{I4}}$ ».

L'œuvre de Vera Frenkel implique aussi une anticipation de l'avenir, une attente. Comme nous l'avons expliqué, l'espace physique du bar permet de conjuguer passé, présent et futur, puisqu'on est en quelque sorte en transit, en déplacement du passé vers le futur, de même que d'un lieu à un autre. Dans un même ordre d'idées, Paul Ricœur écrit de très belle façon :

Seul celui qui peut s'élancer vers le futur par le souci - lequel enveloppe le désir, la crainte, l'attente et la fuite - peut aussi se retourner vers le passé, par la mémoire, le regret, le remords, la commémoration ou l'exécration - et ainsi revenir sur le présent, comme l'aspect du temps où s'échangent l'attente et la mémoire. Sous le premier rapport, le présent est une origine, sous le second, un transit ${ }^{15}$.

C’est réellement cette question du transit qui est importante chez Vera Frenkel, nous l'aurons compris. Et dans ce cas précis où la question du sous-titrage est au centre de nos préoccupations, nous comprenons que ce transit implique une perte pour le témoin - perte de mémoire culturelle, perte d'une langue. Le spectateur est confronté à cela en essayant de suivre le fil d'un récit à l'aide de sous-titres toujours incomplets. Cette perte de sens expérimentée par le spectateur fait donc écho au problème de la perte de langue que Frenkel place au centre de son œuvre. L'artiste inclut effectivement dans ... du transitbar des langues qu'elle qualifie de «discontinuées », comme le yiddish, souvent considéré à tort comme une langue morte. Elle fait ainsi allusion aux langues perdues après une déportation ou un exil forcé - par opposition aux « langues de la bureaucratie », comme le français, l'anglais et l'allemand -, comme ce fut le cas pour ses parents, qui ont dû quitter leur pays d'origine et apprendre une nouvelle langue. L'utilisation du sous-titrage chez Frenkel permet par conséquent de démontrer cette perte. Et accessoirement, comme l'explique Jean Gagnon, le yiddish est souvent considéré comme «un code de transition culturelle, un champ de négociations entre les rêves de l'ancien monde et les réalités du nouveau monde ${ }^{16}$ », une langue devenue formule nécessaire au transfert de référents culturels d'un pays quitté à un pays adopté. Un tel choix choix n'est visiblement pas anodin pour une œuvre axée sur la question du transit.

\footnotetext{
${ }^{14}$ Paul Ricœur, 2003, p. 8.

is Paul Ricœur, Du texte à l'action. Essais d'herméneutique II, Paris, Seuil, 1986, p. 262.

${ }^{16}$ Gagnon et al., 1994, p. 56.
} 


\section{Confrontation et perte de sens chez AnRi Sala}

La deuxième œuvre à l'étude dans le cadre de cette recherche est une œuvre vidéo de style documentaire de l'artiste albanais Anri Sala, Intervista (Finding the Words) (1999). La vidéo d'une vingtaine de minutes est tournée en langue albanaise et sous-titrée en anglais ou en français, selon le contexte d'exposition, mais nous verrons dans les prochaines pages que la question du sous-titrage est ici beaucoup plus engageante que cette utilisation strictement pratique. La vidéo entremêle diverses entrevues, des images d'archives de la guerre civile en Albanie tirées des télévisions italienne et française, de même que des plans mis en scène à divers degrés et permettant de suivre le déroulement d'une enquête menée par l'artiste, lui-même protagoniste du film. Au commencement de la vidéo, l'artiste retrouve — de façon très exagérée, c'està-dire surjouée - un film I6 mm au fond d'une boîte de carton. Sur ce film noir et blanc, nous l'apprenons bien vite, sa mère, Valdet, alors âgée de 32 ans, est interviewée à l'occasion d'un congrès du Parti communiste albanais en 1977, soit environ 20 ans plus tôt. Mais, par malheur, la bande sonore accompagnant la vidéo a été égarée, ce fait témoignant d'une époque où les enregistrements de la télévision nationale se conservaient sur deux bobines distinctes, une pour le son et l'autre pour l'image. L'artiste est alors en mesure de voir sa mère en compagnie du dictateur et d'autres militants, ou être interviewée seule, mais sans pouvoir entendre les propos prononcés à cette occasion.

Cette perte d'une partie de l'archive devient le point de départ de l'œuvre créée par Sala. En effet, dans une espèce de quête à plusieurs volets, l'artiste va prendre contact avec des gens susceptibles de l'aider à retrouver les propos perdus, notamment un ancien preneur de son devenu chauffeur de taxi et des militants ayant également participé au congrès de 1977. Cette quête le conduit dans une école de sourds et muets de Tirana, où il obtient finalement l'aide de ces derniers pour lire sur les lèvres de Valdet afin d'en extraire les propos et de sous-titrer l'entrevue.

Tout comme chez Vera Frenkel et son transitbar, dans Intervista (Finding the Words), nous retrouvons une articulation particulière des temps, alors que passé, présent et futur se rejoignent au sein de la même œuvre. Tout d'abord, comme nous l'avons abordé précédemment, la vidéo de Sala se construit autour d'une enquête et des entretiens menés au présent. L'œuvre a pour point de départ - bien que surgissent parfois des images d'archives et des extraits du film I6 mm de 1977 - le moment où Sala ouvre la boîte de carton et fait la découverte de la bobine. Ce moment se distingue de celui où Sala a réellement trouvé la bobine, puisque ce que l'on voit dans Intervista 
(Finding the Words) n'est sans l'ombre d'un doute qu'un reenactment. De même, l'enquête, qui s'échelonne sur plusieurs semaines, voire plusieurs mois, est, par rapport au moment de la réception du film qui nous la présente, chose du passé. Mais nous la vivons au présent, celui des protagonistes du documentaire dans une Albanie contemporaine. Nous reviendrons plus en détail sur cette question du présent en abordant la question du passé dans la partie suivante de ce travail, puisque nous verrons que des problématiques importantes liées au sous-titrage s'y rapportent.

Dans ce présent de l'enquête que mène Sala pour retrouver les propos perdus de sa mère et parvenir à les sous-titrer, c'est toutefois ce choc avec le passé qui est le plus important, la confrontation de la mère à ses propres paroles. Il faut comprendre qu'à l'époque de l'enregistrement, en 1977, Valdet était partisane du chef du pays Enver Hoxha et militante active des Jeunesses communistes d'Albanie. Avec le temps, le règne d'Hoxha s'est révélé être une véritable dictature, relayée à sa mort en 1985 et n'ayant véritablement pris fin qu'à la chute du régime en 1991. Et, pour reprendre les propos de Nadia Fartas qui a écrit un article de fond sur l'œuvre à l'étude, c'est comme si, dans Intervista, tout cela était « renégocié dans le présent ${ }^{17}$ ». Lorsque son fils lui apporte la cassette sous-titrée de son passage à la télévision nationale dans le cadre du congrès de 1977 (voir la figure 5), Valdet est d'abord confrontée à son passé, et sa première réaction est de renier en bloc le texte qu'elle a sous les yeux, de nier avoir prononcé les propos sous-titrés à l'école de sourds et muets, tellement ils sont éloignés de ses convictions actuelles et ravivent de vieilles blessures (voir la figure 6).
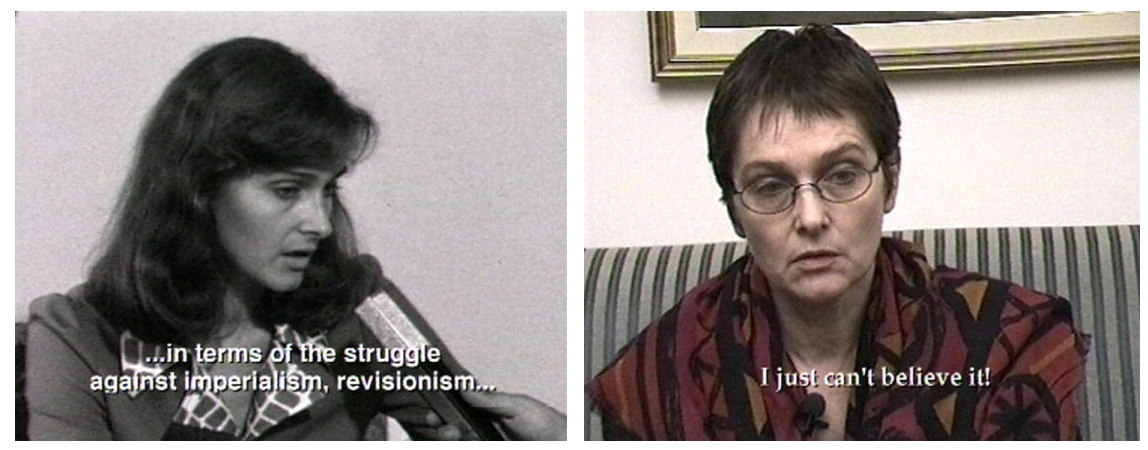

Fig. 5-6. Anri Sala, Intrevista, vidéo et son, 1998, 26 min. Courtoisie de l'artiste et de la Galerie Chantal Crousel, Paris.

(C) Anri Sala/ADAGP P ARIS, 2016

${ }_{17}$ Nadia Fartas, « Temps de la transmission, écarts de la familiarité. Intervista d'Anri Sala », Images Re-vues, $\mathrm{n}^{\circ}$ 9, 20II, http://imagesrevues.revues.org/1629 (consultation le 13 janvier 2017). 
Ce faisant, elle semble vouloir se couper du passé, créer une rupture. Il ne faut toutefois pas se surprendre d'un tel changement de cap, puisque la dictature faisait alors en sorte d'uniformiser le discours. Les questions posées par l'intervieweur à Valdet et aux autres militants étaient construites de façon à obtenir des réponses bien précises, et tout le monde se retrouvait à tenir plus ou moins le même discours, évidemment en faveur d'Hoxha et du Parti communiste albanais, dans une « langue conforme au discours des Jeunesses communistes en Albanie ${ }^{18} \gg$. Mais, paradoxalement, l'objet de la volonté de rupture de Valdet, c'est cette époque de dictature qui, justement, tentait à tout prix de faire table rase et de garder les yeux rivés sur un avenir — obligatoirement meilleur. Comme l'explique toujours Fartas à propos du déni initial de Valdet :

Ce conflit entre l'image et la parole trouve son point critique dans la confusion paradoxale entre deux temps, deux images, celle d'elle-même en 1977 et celle, contemporaine, de la vidéo. Cette confusion s'actualise dans le langage: «C'est absurde ! Non ce n'est pas vrai. Il n'y a aucune structure. Pourtant, je sais articuler ma pensée. [...] Je parle comme ça, moi ?». Dans le présent d'énonciation, Valdet Sala produit une continuité et une indistinction entre le temps et la langue, qui ruinent l'intervalle de vingt ans ${ }^{19}$.

Il y a donc confusion entre passé et présent au moment de la lecture des sous-titres par Valdet, c'est-à-dire dans le présent de l'énonciation. Il est d'autant plus facile pour elle de nier avoir tenu ces propos d'appui au régime d'Hoxha qu'elle ne s'entend pas les dire. Elle-même peut en fait se sentir mise à l'écart de sa propre histoire par un relais dépersonnalisé de ses paroles par le sous-titrage. Mais, dans cette même rencontre avec son fils, Valdet passe du déni à l'acceptation et à l'explication. Elle consent à expliquer le contexte du discours qu'elle tenait alors, évidemment déformé par la toute-puissance de la dictature d'Hoxha (voir la figure 7). La confrontation, l'acceptation et l'explication se produisent ainsi dans le présent, mais ne surviennent qu'au regard d'évènements passés. Valdet elle-même explique alors : « [...] je parle d'une réalité et de mon rapport à cette réalité qui appartient au passé, mais concerne le présent ». Ellemême est consciente de l'impact, sur son présent, de la confrontation du passé qu'implique son processus de remémoration. Alors que chez Vera Frenkel, comme nous l'avons expliqué, le présent faisait en sorte d'enchevêtrer les récits des intervenants et ceux des gens présents au bar pour construire une mémoire partagée universelle (ou eurocentrique, selon les points de vue), ici, c'est la logique de la confrontation qui permet la transmission intergénérationnelle de la mémoire collective des Albanais.

\footnotetext{
18 Fartas, 20II.

I9 Fartas, 20II.
} 


\section{It was real Anri, because we were building.}

Fig. 7. Anri Sala, Intrevista, vidéo et son, 1998, 26 min. Courtoisie de l'artiste et de la Galerie Chantal Crousel, Paris.

(C) Anri Sala/ADAGP PARIS, 2016

Cette confrontation, elle est aussi palpable dans le travail de montage et de resserrement des plans effectué par Sala. Jamais, dans Intervista (Finding the Words), nous ne voyons dans le même cadre une image tirée du film I6 mm de la mère en 1977 et une image de la mère au moment de l'enquête, dans le présent. Nous ne voyons pas non plus les personnes interrogées aujourd'hui dans le même cadre que des images d'archives. Les plans se succèdent sans nous montrer les protagonistes du présent regarder les images du passé que Sala leur amène. Présent et passé sont donc articulés de façon à être en opposition constante. Par ailleurs, lorsque le spectateur regarde le film de Sala, à moins de bien connaître l'albanais, il le comprend inévitablement par les sous-titres anglais ou français. Ces sous-titres « utilitaires » viennent par moments réunir les deux temporalités en une confrontation. Le sous-titrage des propos prononcés par Valdet en 1999 vient s'apposer de manière fortuite sur des images de 1977, alors que le film n'est en aucun cas conçu ainsi. Quand Valdet dit «I'm 52 » («J'ai 52 ans »), en 1999, ces propos font partie du présent de l'énonciation abordé précédemment, bien que ces mots sous-titrés apparaissent sur des images de 1977, se 
succédant au montage. Le sous-titrage utilitaire vient ici contaminer l'image d'archives, faisant en sorte de réconcilier de manière involontaire présent et passé. Mais il n’y a pas que le sous-titrage qui vient réconcilier les deux temporalités que tout tend à opposer. Au-delà de cette réunion fortuite du passé et du présent par un détail technique, c'est Anri Sala lui-même, passeur entre les temps, qui crée les hétérogénéités. Sans son enquête pour retrouver les propos perdus et son travail pour sous-titrer le film $16 \mathrm{~mm}$, la confrontation n'aurait pas lieu, le processus de remémoration ne s'enclencherait pas. En adoptant ce rôle, Sala emprunte une trajectoire similaire à celle du wagon-bar, qui fait cheminer du passé au présent au futur, et décloisonne l'archive.

Comme dans l'œuvre de Vera Frenkel, il est aussi question de perte chez Anri Sala. En effet, tout au long de son enquête, ce dernier tente de retrouver un élément du passé et, dans les faits, il va y arriver. Que cela plaise à Valdet ou non, l'artiste va réellement retrouver ce qui a été dit au congrès du Parti communiste albanais de 1977 et le transposer en sous-titres. Mais, malgré toute l'attention portée à la fidélité des soustitres par rapport aux propos tenus, nous nous rendons bien compte que, même en ayant en quelque sorte « retrouvé le passé », il y a une perte énorme. Le discours retrouvé, c'est un discours faussé, manipulé par la dictature. C'est une construction. Ce que la traductrice de l'école de sourds et muets de Tirana recompose et décompose, ce sont «les paroles d'une langue qui combine ou, du moins, ne distingue pas, propagande et militantisme $e^{20} \gg$. Sala récupère donc le texte sans en retrouver le sens véritable, en quelque sorte. Ce qu'il retrouve, c'est «l'inanité de [...] la syntaxe du totalitarisme ${ }^{2 I} \gg$, une suite de propos qui ne veulent pas dire grand-chose et qui déroutent sa mère.

Cette perte de sens, si nous mettons de côté un instant ce motif de la dictature, prend aussi naissance dans des questions plus techniques. En effet, alors que la traduction et le sous-titrage, comme chez Vera Frenkel, occasionnent d'inévitables pertes, la transcription des propos d'une technique à une autre agit de façon semblable, presque à la manière d'un téléphone arabe. Lors de la visite de son fils, Valdet semble remettre en question la validité de la lecture sur les lèvres de l'interprète de même que la convertibilité de ses propos d'une technique à une autre - du film $16 \mathrm{~mm}$ à la cassette vidéo. Sala doit même lui assurer qu'il n'a en aucun cas édité la cassette ou la bande du film pour manipuler son discours. Comme l'explique l'auteur Marco Purpura dans un texte sur la migration albanienne en Italie :

\footnotetext{
${ }^{20}$ Fartas, $201 \mathrm{2}$.

${ }^{21}$ Larisa Dryansky, « Anri Sala : l'art des interférences », 20/27 revue de textes critiques sur l'art, $\mathrm{n}_{3}{ }_{3}, 2009$, p. 177.
} 
La résistance de Valdet implique qu'il est possible de se perdre dans la transcription linguistique et que la traduction peut aussi se perdre dans la conversion technique. [...] Cela est aussi applicable à l'expérience du spectateur et est amplifié par la conversion subséquente par Sala de sa vidéo au format DVD afin d'être projetée en boucle dans l'espace du musée ${ }^{22}$.

Nous comprenons ainsi que la perte peut subvenir à plusieurs étapes de l'enquête de Sala, dans cette chaîne de conversions techniques, c'est-à-dire qu'au départ, le film $16 \mathrm{~mm}$ retrouvé aurait sans doute eu besoin d'une restauration pour retrouver sa qualité d'image initiale, alors qu'un transfert sur cassette vidéo n'a sans doute fait qu'amplifier les défauts déjà présents sur le film défraîchi. L'interprète de l'école des sourds et muets de Tirana se basant sur une copie de qualité moindre pour son travail de sous-titrage, il devient difficile de bien lire sur les lèvres, de saisir toutes les subtilités. Le spectateur n'ayant ensuite accès qu'à la version du film en format DVD pour le musée, après coupures et montage, il apparaît évident que les pertes sont inévitables.

Comme nous l'avons expliqué précédemment, chez Anri Sala comme chez Vera Frenkel, passé, présent et futur s'articulent au sein d'une même œuvre dans un processus de transmission de mémoire. Dans Intervista (Finding the Words), le futur est également convoqué. Déjà en 1977 , les propos que tient Valdet au congrès du Parti communiste albanais sont des propos sur l'avenir de l'Albanie. Ce sont des propos remplis d'espoir d'un avenir radieux pour le pays. Mais, ici encore, une confrontation survient entre la vision du futur qu'avait Valdet en 1977 et celle qu'elle a dans le présent. Après avoir été confrontée au discours qu'elle tenait vingt ans plus tôt, celle-ci explique que son point de vue n'est plus du tout le même. Dans une scène du film de i999, elle tient d'ailleurs ces propos très éloquents :

J'ai peur parce que je ne vois pas d'issue pour l'avenir. Je ne comprends plus ce qui se passe. J'ai peur. Tout est confus. Quand je parle de l'avenir, je pense à tout ce qui est proche de moi, mais aussi au destin du pays. Les évènements récents ont brisé beaucoup d'espoirs. [...] Si ce pays a un avenir, vous en aurez un aussi. Mais s'il n'en a pas, vous n'en aurez pas non plus.

Dans cet écart de vingt ans, la vision du futur de l'ancienne membre des Jeunesses communistes d'Albanie passe donc de l'espoir et de l'insouciance à la peur et à la confusion. Mais, dans cet aveu d'une crainte en l'avenir, Valdet tend également un pont entre mémoire personnelle et mémoire collective, et entre futur personnel et futur

${ }^{22}$ Marco Purpura, «Transmedia Memory of Albanian Migration in Italy: Helidon Gjergii's, Adrian Paci's, and Anri Sala's Moving-Image Installations », California Italian Studies, vol. 2, $\mathrm{n}^{\mathrm{o}} \mathrm{I}, 2 \mathrm{II}$, p. 20. (Notre traduction.) 
collectif. Alors que Vera Frenkel crée une sorte de narration collective en travaillant sur l'enchevêtrement des récits, réunissant une somme de mémoires personnelles dans un même présent et participant ainsi à la construction d'une mémoire collective, les propos de Valdet tendent également à osciller entre les deux niveaux de mémoire et à mettre de l'avant deux types de futur pour son pays. Ici, la mère de l'artiste confronte effectivement l'avenir du pays à celui de chacun de ses habitants pris séparément. Comme l'explique Mark Godfrey dans un ouvrage consacré à Anri Sala :

[celui-ci] aborde l'histoire publique par la mémoire personnelle - et les écarts de mémoire - de sa mère. Ce faisant, il reconnaît l'impossibilité d'une «approche objective » d'un sujet historique et admet que tout le monde a son rôle à jouer dans le passé trouble de l'Albanie. Il suggère également que la confrontation historique en général est un constant processus plutôt qu'un dossier classé, un processus par lequel chaque crise apporte avec elle autant de nouvelles crises que de solutions ${ }^{23}$.

En cela, Anri Sala rejoint Vera Frenkel, qui affirmait que le but d'un travail artistique comme le sien n'est pas de combler les trous de la mémoire, car cela serait impossible. Il reconnaît également qu'essayer de corriger le récit du passé afin d'obtenir une histoire objective est un projet vain, puisque, comme l'explique Ricœur, « [c'est] une illusion de croire que les énoncés factuels puissent satisfaire à l'idée de l'irreprésentable, comme si des faits pouvaient par la vertu de leur présentation littérale être dissociés de leur représentation en forme d'évènements dans une histoire ${ }^{24} \gg$. La somme des récits, aussi détaillés et factuels soient-ils, reste une structure mouvante, et de chaque crise émaneront toujours de nouvelles crises à ajouter à l'histoire.

Nous comprenons donc que, dans les deux œuvres à l'étude, soit ...du transitbar (1992) de Vera Frenkel et Intervista (Finding the Words) (1999) d'Anri Sala, l'utilisation du sous-titrage comme matériau artistique permet en quelque sorte de créer une articulation des temps où présent, passé et futur cohabitent et s'entremêlent. Dans les deux cas, le temps présent semble être un temps performatif dans le cadre duquel une série d'actions peuvent être accomplies, notamment la remémoration (Valdet Sala puisant dans ses souvenirs afin de recoller les morceaux d'une histoire ambiguë ou les intervenants de l'œuvre de Vera Frenkel partageant leur expérience de

\footnotetext{
${ }^{23}$ Mark Godfrey, Hans Ulrich Obrist et Liam Gillick, Anri Sala, Londres, Phaidon, 2006, p. 36, notre traduction.

${ }^{24}$ Paul Ricœur, 200o, p. 333.
} 
déplacement volontaire ou forcé) ou encore l'énonciation (Valdet Sala niant avoir tenu certains propos vingt ans auparavant). Pour Jean Gagnon, d'ailleurs, le temps présent du piano-bar de Frenkel est aussi une « catégorie temporelle de l'agir et du souffrir ${ }^{25}$ », « l'espace où le traumatisme naît au monde, à la langue et à la représentation grâce à l'articulation de la mémoire ${ }^{26} \gg$.

Ce présent permet également une réactualisation du passé par une intervention de la mémoire. En effet, lorsque réactualisées, les histoires du passé de tout un chacun se conjuguent afin de former une narration commune, en marge de la narration personnelle toujours subsistante. Chez Frenkel comme chez Sala, nous assistons en somme à un jeu de renvois entre mémoire personnelle et mémoire collective, de même qu'entre futur personnel et futur collectif. Les deux œuvres prennent effectivement comme objet une relation intime au passé, mais mettent aussi de l'avant des évènements plus directement ancrés dans la « grande histoire », celle des déportations et des exils chez Frenkel, celle du communisme albanien chez Sala. Toutefois, au-delà de ce retour direct sur le passé, nous voyons que l'utilisation du sous-titrage complexifie l'articulation des temps en créant une perte de sens ou une confrontation. Sans ce dispositif technique mis en place par les artistes à l'étude, Valdet n'aurait pas été confrontée à son passé de jeune militante et les clients du piano-bar de Frenkel n'auraient pas, par l'expérience du manque linguistique, été sensibilisés à la perte culturelle engendrée par le déplacement de certaines populations.

Considérant les problématiques liées à l'exposition de l'art vidéo en contexte muséal, nous voyons finalement qu'une transformation de la simple fonction utilitaire du sous-titrage en un vecteur d'opérativité créatrice est possible. Chez Frenkel comme chez Sala, une utilisation subversive du sous-titrage permet de mettre de l'avant des questions liées à la mémoire, à la transmission et à la réécriture d'une histoire personnelle ou collective, et de sortir de la simple question de la fidélité en traduction. Alors que nombreux sont les cas d'œuvres dont l'expérience d'origine est complètement transformée, voire oblitérée, par une réexposition avec sous-titrage, les possibilités qu'offre un tel outil sont multiples, autant chez les commissaires, libres d'ajouter une strate narrative au sous-texte de leurs expositions, que chez les artistes, jouissant d'une agentivité nouvelle par cette mise de l'avant des défauts de traduction et de compréhension.

\footnotetext{
25 Gagnon et al., 1994, p. 22.

${ }^{26}$ Ibid., p. 53.
} 


\title{
Des problématiques de présentation de l'art vidéo à l'agentivité créatrice : sous-titrage et articulation des temps chez Anri Sala et Vera Frenkel
}

\author{
Maude lefebvre, Université du QuÉBEc À Montréal
}

\section{RÉSUMÉ}

L'exposition de l'art vidéo en contexte muséal donne souvent lieu à des problématiques liées entre autres à la question du sous-titrage dans un but de diffusion multilingue. L'utilisation d'un tel moyen technique occasionne dans bien des cas des pertes de sens importantes, voire l'effacement total de la dimension sonore, mais peut également produire un décloisonnement des médiums et une simultanéité des expériences. Certains artistes utilisent quant à eux le sous-titrage comme matériau artistique à part entière, comme la Canadienne d'origine slovaque Vera Frenkel et l'Albanais Anri Sala.

\section{ABSTRACT}

Exhibiting video art in a museum setting often raises questions related to the practice of subtitling for the purposes of multilingual dissemination of the artwork. In many cases, the use of such technical means results in a significant loss of meaning or even the complete obliteration of the sound dimension of the work, although it can also give rise to a decompartmentalization of artistic mediums and a simultaneity of experiences. Some artists, for their part, use subtitling as a full artistic medium-for instance, the Canadian artist of Slovak origin Vera Frenkel and the Albanian Anri Sala.

\section{NOTE BIOGRAPHIQUE}

Maude LefebVRe est étudiante à la maîtrise en histoire de l'art à l'Université du Québec à Montréal. Ses recherches portent sur le sous-titrage et l'exposition de l'art vidéo en contexte muséal. Elle a écrit pour plusieurs revues Web dédiées aux arts visuels et a collaboré à l'édition de l'ouvrage Questionner l'avenir. Réflexions sur la réactualisation de la Biennale de Montréal (Éditions d'art Le Sabord, 20I5). Elle travaille également dans le milieu du sous-titrage pour malentendants. 